\title{
DETERMINACIÓN DE FACTORES DE RIESGO DE AGITACIÓN POST-ANESTESICA EN PACIENTES PEDIATRICOS
}

González Arellano Alejandro ${ }^{1}$, Ibacache Figueroa Mauricioํㅜㄹ Puga Aravena Valentina ${ }^{2}$, Carmona B. Javiera ${ }^{1}$, De la Fuente L. Natalia ${ }^{2}$, Zamora Heloc Maximiliano ${ }^{1}$

1 División de Anestesia, Pontificia Universidad Católica de Chile.

2 Hospital Clínico de La Florida.

Introducción: La agitación post-anestésica (APA) ocurre en 10 a $80 \%$ de los niños. No tiene etiología definida, pero hay descritos factores relacionados y criterios diagnósticos. Durante la agitación los pacientes pediátricos se pueden autolesionar o lesionar a terceros. Identificar factores perioperatorios de riesgo de APA sería útil para prevenir su ocurrencia.

Objetivo General: Identificar factores perioperatorios de riesgo que se asocian a la ocurrencia de APA en pacientes pediátricos sometidos a anestesia general con sevofluorano.

Material y Métodos: Estudio prospectivo, aprobado por ética, en pacientes de 2-12 años, ASA I-II, sometidos a cirugía electiva bajo anestesia general con sevofluorano. Se excluyeron: cirugía cardíaca, neurocirugía, procedimientos radiológicos; pacientes de unidades intensivas, con discapacidad mental, operados u hospitalizados en 3 meses previos. En unidad preoperatoria se registraron: variables demográficas del paciente y padre; ansiedad del paciente con Escala de Ansiedad Preoperatoria Yale Modificada (mYPAS); ansiedad del padre con Escala Likert
Auto-reporte. En pabellón se registraron: ansiedad del paciente (mYPAS) y del padre (Likert); comportamiento del paciente durante inducción con Lista Comportamiento Inducción; drogas y técnica de anestesia y de analgesia postoperatoria; incidentes; cirugía realizada; tiempos quirúrgico y anestesia; APA al despertar con Escala Agitación Post-Anestésica Pediátrica (PAED) En recuperación se registraron, cada 15 minutos durante 2 horas: APA (PAED); dolor con escala CHIPPS o EVA según corresponda. Análisis estadístico: descriptivo; correlación de Pearson o Spearman; regresión logística; significativa $\mathrm{p}$ $<0,05$.

Resultados: Se reclutaron 211 pacientes, cuyas variables demográficas se muestran en tabla 1. La incidencia de ansiedad preoperatoria y en pabellón fue $29,2 \%$ y $41 \%$ en los pacientes y $51 \%$ y $53,3 \%$ en los padres respectivamente; hubo una correlación positiva en ansiedad preoperatoria entre padres y pacientes $(p<0,007)$. La incidencia de APA al despertar y en recuperación fue $36,7 \%$ y $32,1 \%$ respectivamente. El $31 \%$ de los pacientes tuvo dolor postoperatorio leve a

Tabla 1. Variables demográficas

\begin{tabular}{|llc|}
\hline Edad (años) & & $6,07+2,79$ \\
Género (\%) & Hombres & 65,9 \\
& Mujeres & 34,1 \\
ASA (\%) & I & 77,6 \\
& II & 22,4 \\
Tipo de cirugía (\%) & & 53,1 \\
Circunsición, herniorrafia, orquidopexia & & 27,8 \\
Adenoamigdalectomia & & 10,5 \\
Adenoidectomia c/punción timpánica & & 3,3 \\
Digestiva, urológica & & 3,3 \\
Traumatológica & & 2 \\
Cabeza y cuello & & 2 \\
\hline
\end{tabular}


moderado. La regresión logística identificó los siguientes factores asociados con APA: la edad del paciente $(\mathrm{p}=0,000)$, la adenoamigdalectomía $(\mathrm{p}=0,013)$ y el dolor postoperatorio $(\mathrm{p}=$ $0,000)$. No hubo asociación de la APA con la ansiedad del paciente y/o del padre, antecedente de cirugías previas, el comportamiento del paciente durante la inducción anestésica o la duración de la cirugía.

Conclusiones: La APA se presentó en un tercio de nuestros pacientes, incidencia que concuerda con lo reportado. De los factores estudiados y de los que han sido descritos en la literatura solo la edad del paciente, la cirugía de adenoamigdalas y la presencia de dolor postoperatorio muestran una clara relación con la ocurrencia de APA. La presencia de alguno de estos factores permitirían predecir y prevenir la ocurrencia de APA en pacientes pediátricos sometidos a anestesia general con sevofluorano. 